Physics

Physics Research Publications

Purdue University

Year 2005

\title{
Andreev reflection and pair-breaking effects at the superconductor/magnetic semiconductor interface
}

R. P. Panguluri*

X. Liu**
K. C. $\mathrm{Ku}^{\dagger}$

J. K. Furdyna ${ }^{\dagger \dagger}$

$\begin{array}{ll}\text { N. Samarth } & \text { B. Nadgorny }\end{array}$

N. Samarth ${ }^{\S} \quad$ B. Nadgorny
T. Wojtowicz ${ }^{\ddagger}$

Y. B. Lyanda-Geller

This paper is posted at Purdue e-Pubs.

http://docs.lib.purdue.edu/physics_articles/201 


\title{
Andreev reflection and pair-breaking effects at the superconductor/magnetic semiconductor interface
}

\author{
R. P. Panguluri, ${ }^{1}$ K. C. Ku, ${ }^{2}$ T. Wojtowicz, ${ }^{3,4}$ X. Liu, ${ }^{3}$ J. K. Furdyna, ${ }^{3}$ Y. B. Lyanda-Geller, ${ }^{5}$ N. Samarth, ${ }^{2}$ and B. Nadgorny ${ }^{1}$ \\ ${ }^{1}$ Department of Physics and Astronomy, Wayne State University, Detroit, Michigan 48201, USA \\ ${ }^{2}$ Department of Physics and Materials Research Institute, The Pennsylvania State University, \\ University Park, Pennsylvania 16802, USA \\ ${ }^{3}$ Department of Physics, University of Notre Dame, Notre Dame, Indiana 46556, USA \\ ${ }^{4}$ Institute of Physics, Polish Academy of Sciences, 02-668 Warsaw, Poland \\ ${ }^{5}$ Department of Physics, Purdue University, West Lafayette, Indiana 47907, USA
}

(Received 3 November 2004; published 9 August 2005)

\begin{abstract}
We investigate the applicability of spin-polarization measurements using Andreev reflection in a pointcontact geometry in heavily doped dilute magnetic semiconductors, such as (Ga,Mn)As. Although we observe conventional Andreev reflection in nonmagnetic $(\mathrm{Ga}, \mathrm{Be})$ As epilayers, our measurements indicate that in ferromagnetic $(\mathrm{Ga}, \mathrm{Mn})$ As epilayers with comparable hole concentration the conductance spectra can only be adequately described by a broadened density of states and a reduced superconducting gap. We suggest that these pair-breaking effects stem from inelastic scattering in the metallic impurity band of (Ga,Mn)As and can be explained by introducing a finite quasiparticle lifetime or a higher effective temperature. For $(\mathrm{Ga}, \mathrm{Mn}) \mathrm{As}$ with $8 \% \mathrm{Mn}$ concentration and $140 \mathrm{~K}$ Curie temperature we evaluate the spin polarization to be $83 \pm 17 \%$.
\end{abstract}

\section{DOI: 10.1103/PhysRevB.72.054510}

The advance of semiconductor spintronics has revived a long-standing interest in understanding the coupling of charge and spin in semiconductors. ${ }^{1}$ Ferromagnetic semiconductors ${ }^{2,3}$ are of central importance to semiconductor spintronics because they have a conductivity compatible with that of conventional semiconductors and the potential for a high intrinsic spin polarization, thus, providing promising conditions for efficient spin injection into conventional semiconductors. In this context the ferromagnetic semiconductor (Ga,Mn)As with Curie temperatures routinely reproducible in the range of $140 \lesssim T_{C} \lesssim 170 \mathrm{~K}$ (Refs. 4 and 5) stands out as a well-studied model system. ${ }^{2,3}$ Furthermore, (Ga,Mn)As has been successfully incorporated into a variety of spininjection and spin-transport devices. ${ }^{6}$ Although measurements of the carrier (hole) spin polarization in this material are immediately relevant to contemporary efforts in semiconductor spintronics, systematic experiments probing this important quantity are just beginning. ${ }^{7}$

Spin-polarization measurements can be carried out using the tunneling geometry in superconductor (S)/insulator (I)/ ferromagnet (FM) structures $;^{8}$ however, attempts to use this technique in $(\mathrm{Ga}, \mathrm{Mn})$ As have thus far been unsuccessful. ${ }^{7}$ In spite of some theoretical problems, ${ }^{9,10}$ Andreev reflection $(\mathrm{AR})$ in a $\mathrm{FM} / \mathrm{S}$ contact $^{11,12}$ provides a viable alternative to tunneling for measurements of the spin polarization $(P)$ in a variety of materials, including ferromagnetic metals and metallic oxides. ${ }^{13}$ Recently, AR measurements in planar junctions of $\mathrm{Ga} /(\mathrm{Ga}, \mathrm{Mn})$ As have estimated a value of $P$ $\sim 85 \%$ for samples with $5 \% \mathrm{Mn}$ and $T_{C}=65 \mathrm{~K} .^{7}$ Despite extensive attempts to make epitaxial $\mathrm{S} /(\mathrm{Ga}, \mathrm{Mn})$ As planar junctions with a variety of superconductors, useful data has been obtained only in a limited number of $\mathrm{Ga} /(\mathrm{Ga}, \mathrm{Mn}) \mathrm{As}$ samples, ${ }^{7}$ suggesting extreme sensitivity to the nature of the heterointerface. Additionally, the planar geometry has unavoidable limitations imposed by constraints on the materials growth, limiting the postgrowth modifications of the sample
PACS number(s): 74.45.+c, 72.25.Mk, 72.25.Dc

characteristics $^{14}$ and thus restricting AR measurements to (Ga,Mn)As samples with relatively low Curie temperatures $\left(T_{C} \sim 65 \mathrm{~K}\right)$. Finally, the conductance spectra in Ref. 7 have been explained using a distribution of the energy gaps in the $\mathrm{Ga}$ superconducting film. An alternative interpretation of this data has also been suggested, involving a distortion of the density of states in a superconductor. ${ }^{15}$ In this context, the measurements of spin polarization in $(\mathrm{Ga}, \mathrm{Mn}) \mathrm{As}$ in the conventional point contact Andreev reflection (PCAR) geometry are vital for both extending the range of sample parameters as well as for resolving different interpretation of the data.

In this paper, we use PCAR to evaluate $P$ in $(\mathrm{Ga}, \mathrm{Mn}) \mathrm{As}$ epitaxial layers with a high Curie temperature, $T_{C}=140 \mathrm{~K}$. In order to develop a reliable interpretation of our study of (Ga,Mn)As, we first apply the PCAR technique to a nonmagnetic analog of (Ga,Mn)As- $(\mathrm{Ga}, \mathrm{Be}) \mathrm{As}$ - with doping concentrations similar to those in the ferromagnetic semiconductor $\left(p \sim 10^{21} \mathrm{~cm}^{-3}\right)$. Our PCAR studies of $(\mathrm{Ga}, \mathrm{Be}) \mathrm{As}$ yield the data that is well described by a conventional weak coupling Blonder-Tinkham-Klapwijk (BTK) model. ${ }^{16}$ In contrast, the PCAR experiments in ferromagnetic (Ga,Mn)As cannot be described by a simple BTK model modified for the spin-polarized case. ${ }^{17}$ The (Ga,Mn)As data indicate a significant broadening of the density of states (DOS) accompanied by a reduction of the bulk superconducting gap $\Delta_{b}$. We note that these observations are not an intrinsic characteristic of ferromagnetic semiconductors: for instance, PCAR measurements of (In,Mn)Sb-a higher mobility ferromagnetic semiconductor-consistently yield the bulk superconducting gap with no DOS broadening. ${ }^{18}$ This suggests that our observations in $(\mathrm{Ga}, \mathrm{Mn})$ As stem from inelastic scattering in a low-mobility ferromagnetic semiconductor.

Since the pioneering work of Kastalsky et al., ${ }^{19}$ most studies of AR in semiconductors have been carried out in a two-dimensional (2D) geometry. This is not surprising, as serious problems are anticipated for AR experiments in a 

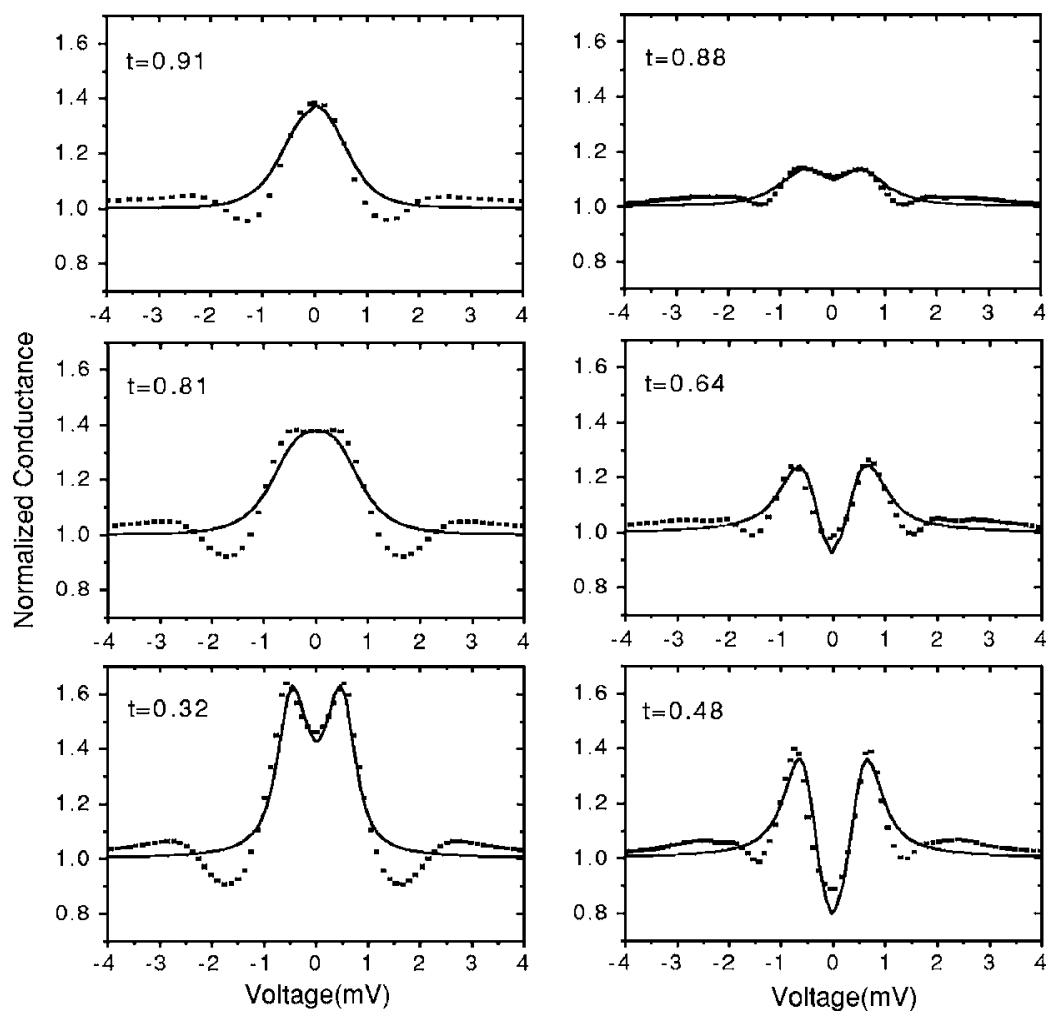

FIG. 1. Two $\mathrm{Sn} /(\mathrm{Ga}, \mathrm{Be})$ As contacts at different reduced temperatures $t=T / T_{c}$ analyzed with the model of Ref. 17. Left panel: Contact resistance $R_{c}=35 \Omega, Z \sim 0.45$; right panel: $R_{c}=28 \Omega$, $Z \sim 0.8$. A small dip above $\Delta$ is due to the proximity effect. superconductor-semiconductor $(\mathrm{S} / \mathrm{Sm})$ junction in a threedimensional (3D) geometry because of the high resistivity of semiconductors at low temperatures and the presence of a Schottky barrier in most S/Sm contacts. The Schottky barrier fundamentally limits the accuracy of spin-polarization measurements in ferromagnetic semiconductors by strongly decreasing the probability of AR. To avoid both of these problems, we use heavily doped $(\mathrm{Ga}, \mathrm{Be}) \mathrm{As}$ and $(\mathrm{Ga}, \mathrm{Mn}) \mathrm{As}$ semiconductors with metallic-type conductivity and, thus, thin Schottky barriers, which make highly transparent S/Sm junctions. $^{20}$

The models of ferromagnetism in $(\mathrm{Ga}, \mathrm{Mn})$ As discussed in the literature invoke either free valence hole $e^{2,21}$ or impurity bands. $^{22}$ Both $\mathrm{Mn}$ and Be are nominally acceptors, with binding energies of 113 and $28 \mathrm{meV}$, respectively. As a result, at low doping concentrations transport in both $(\mathrm{Ga}$, $\mathrm{Be}) \mathrm{As}$ and $(\mathrm{Ga}, \mathrm{Mn}) \mathrm{As}$ at our characteristic experimental temperature $T=1 \mathrm{~K}$ must be described by impurity bands. At high doping levels of $N \sim 10^{21} \mathrm{~cm}^{-3}$ we assume that impurity disorder yields spacial modulation near the top of the valence band, which results in a metallic state with "ballistic" propagation through the contact for $(\mathrm{Ga}, \mathrm{Be}) \mathrm{As}$, and "diffusive" propagation for $(\mathrm{Ga}, \mathrm{Mn}) \mathrm{As}$. Note that the energy scale determined by kinetic and potential energies $e^{2} N^{1 / 3} / \varepsilon$ and $\hbar^{2} N^{2 / 3} / 2 m$, respectively, is of the order of $100 \mathrm{meV}$, where $\varepsilon$ is the dielectric constant and $m$ is the effective mass. This is especially important for (Be,Ga)As, which behaves as a conventional heavily doped semiconductor in which the valence band is modulated by the impurity potential.

A number of $230 \mathrm{~nm}$ thick $(\mathrm{Ga}, \mathrm{Be})$ As samples with hole concentrations $p=8 \times 10^{20} \mathrm{~cm}^{-3}$ and $p=5 \times 10^{20} \mathrm{~cm}^{-3}$ were grown by low-temperature (LT) molecular-beam epitaxy (Riber 32 R\&D) on semi-insulating (001) GaAs substrates.
The $15 \mathrm{~nm}$ thick (Ga,Mn)As samples with a Mn composition of $8 \%$ were grown in an EPI 930 system on $n+$, epi-ready (001) GaAs substrates using conditions described elsewhere. ${ }^{4}$ Postgrowth annealing of the $(\mathrm{Ga}, \mathrm{Mn})$ As samples at $250{ }^{\circ} \mathrm{C}$ yielded $T_{C}=140 \mathrm{~K}$ and a resistivity $\rho \sim 2$ $\mathrm{m} \Omega \mathrm{cm}$ at $4.2 \mathrm{~K} .{ }^{4}$ A point contact is established between the sample and a mechanically polished Sn tip. Conductance $(d I / d V)$ curves were measured with the standard lock-in technique, as described, in detail, in Ref. 23, allowing us to monitor the characteristics of same point contact from $\sim 1 \mathrm{~K}$ to the critical temperature $T_{c}=3.7 \mathrm{~K}$ of the $\mathrm{Sn}$ tip.

To study the properties of AR in nonmagnetic semiconductors, we have measured a series of temperature dependencies for a large number of different $\mathrm{Sn} /(\mathrm{Ga}, \mathrm{Be}) \mathrm{As}$ point contacts. In Fig. 1, we show the evolution of $d I / d V$ for two typical contacts in a sample with a hole concentration $p=8$ $\times 10^{20} \mathrm{~cm}^{-3}$ and a residual resistivity $\rho \sim 150 \mu \Omega \mathrm{cm}$.

Each of the $d I / d V$ curves is analyzed independently using the model of Ref. 17, with the interface transparency of a contact characterized by a dimensionless parameter $Z$. In this analysis, we use the measured physical temperature of the contact and the corresponding value of the Bardeen-CooperSchrieffer (BCS) gap $\Delta_{b}$. This procedure results in a range of $Z$ for different contacts $0.4<Z<0.8$, with $Z$ practically temperature independent for a given contact. The resistivity and measured carrier concentration for $(\mathrm{Ga}, \mathrm{Be}) \mathrm{As}$ yield a meanfree path $l \sim 10 \mathrm{~nm}$; this is comparable to the contact size $d \sim 10 \mathrm{~nm}$ and suggests that the measurements occur in the ballistic transport regime. Although complications may arise from the need to match wave functions of different symmetry from $(\mathrm{Ga}, \mathrm{Be})$ As and $\mathrm{Sn},{ }^{9}$ we will attempt to describe the system phenomenologically following Refs. 16 and 24. For $(\mathrm{Ga}, \mathrm{Be}) \mathrm{As}$ we assume that the impurity and valence bands 


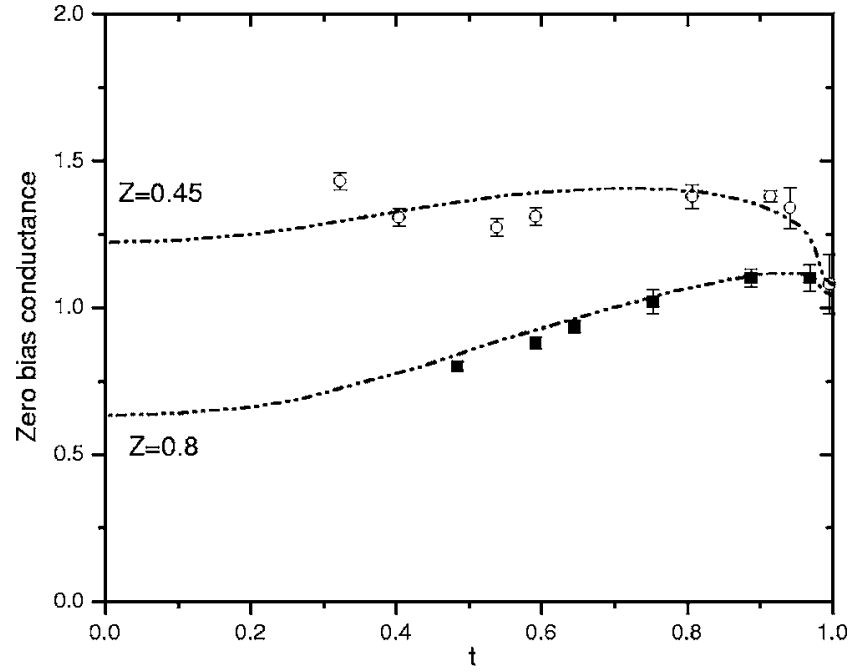

FIG. 2. Zero-bias conductance for the two contacts shown in Fig. 1. Dashed lines show the exact results obtained from the BTK model for $Z=0.45$ and $Z=0.8$, respectively.

overlap, and thus we can still use light and heavy holes to estimate the minimum $Z$ values, $Z=\left[(r-1)^{2} / 4 r\right]^{1 / 2}$, which are due to the Fermi velocity mismatch $r$ between the superconductor $(\mathrm{Sn})$ and $(\mathrm{Ga}, \mathrm{Be}) \mathrm{As}, r=v_{\mathrm{Sn}} / v_{\mathrm{GaAs}}$. Simple estimates of $r$ for light and heavy holes, $r_{l} \sim m_{l h}\left(\left(n_{\mathrm{Sn}} /\right.\right.$ $\left.\left.n_{\mathrm{GaAs}}\right)^{1 / 3}\right) \sim 1.7$ and $r_{h} \sim m_{h h}\left(\left(n_{\mathrm{Sn}} / n_{\mathrm{GaAs}}\right)^{1 / 3}\right) \sim 4$, result in $Z_{h h} \sim 0.8$ and $Z_{l h} \sim 0.3$, in good agreement with the $Z$ values obtained from analyzing $d I / d V$ curves. The experimental zero-bias conductance for the two contacts shown in Fig. 1 and the two corresponding curves obtained independently from the BTK model with $Z=0.45$ and $Z=0.8$ are shown in Fig. 2.

The surprisingly good agreement between the data and the BTK model indicate the "canonical" AR, typically observed in all-metal systems. ${ }^{16}$ Similar results were obtained for $(\mathrm{Ga}$, Be)As with $p=5 \times 10^{20} \mathrm{~cm}^{-3}$ and for (In,Be)Sb. ${ }^{18}$ These results, in conjunction with the estimates of the $Z$-values based on the Fermi velocity mismatch, suggest that the use of highly doped semiconductors can minimize the role of the Schottky barrier in these measurements. Simple estimates yield the Schottky barrier thickness in this system of the order of several angstroms, confirming these conclusions. This is also consistent with the experimentally observed symmetric and linear $I-V$ characteristics above $\Delta_{b}$.

After demonstrating that we can thoroughly understand the PCAR measurements in the heavily doped $(\mathrm{Ga}, \mathrm{Be}) \mathrm{As}$, we now turn to the ferromagnetic semiconductor $(\mathrm{Ga}, \mathrm{Mn}) \mathrm{As}$ with comparable carrier concentration. A number of Sn contacts with $(\mathrm{Ga}, \mathrm{Mn})$ As epilayers have been investigated. Qualitatively, all the contacts appear similar and exhibit a zero-bias conductance that is significantly smaller than the conductance at $V \gg \Delta / e$, suggesting a high spin polarization in $(\mathrm{Ga}, \mathrm{Mn})$ As. However, analyzing the data is much more difficult compared to both the nonmagnetic case of $(\mathrm{Ga}$, $\mathrm{Be}) \mathrm{As}$ and the magnetic case of $(\mathrm{In}, \mathrm{Mn}) \mathrm{Sb}^{18}$ We find that AR in $(\mathrm{Ga}, \mathrm{Mn})$ As does not fit a model of Ref. 17, as all the experimental curves show a strong broadening of the DOS and reduction of the superconducting gap (see Fig. 3).

Our results on $(\mathrm{Ga}, \mathrm{Mn}) \mathrm{As}$ as well as $(\mathrm{In}, \mathrm{Mn}) \mathrm{Sb}^{18}$ indicate that transport processes occurring in the semiconductor are most likely responsible for the observed effects. ${ }^{25}$ In the ballistic regime $l>d$, typical for most AR experiments, the conductance is determined by the transparency of the FM/S interface with the bulk superconducting gap $\Delta_{b}$. For $(\mathrm{In}, \mathrm{Mn}) \mathrm{Sb},{ }^{18}$ our estimates show that holes are all in the ballistic regime. Thus the ( $\mathrm{In}, \mathrm{Mn}) \mathrm{Sb}$ data are easily interpreted in terms of a ballistic model. ${ }^{17}$ In strong contrast, transport in the $(\mathrm{Ga}, \mathrm{Mn})$ As impurity bands is diffusive, with $l \sim 2 \mathrm{~nm}$. Although diffusive transport in AR experiments in metals can be described by conventional theory, ${ }^{17}$ in $(\mathrm{Ga}$, Mn)As with $d \sim 150 \mathrm{~nm}$ the holes spend significant time $t^{*}$ $\sim d^{2} / D \sim 10^{-10} \mathrm{~s}$ within the contact area, where $D$ is the diffusivity. Hence the holes experience enhanced inelastic and spin-flip scattering, ${ }^{26,27}$ with $t^{*}$ comparable to the hole scattering time $\tau_{\varepsilon} \sim 10^{-9}-10^{-10} \mathrm{~s}$ due to acoustic phonons and hole-hole interaction. However, the observed broadened DOS and reduced $\Delta_{b}$ both require processes with shorter characteristic times $\sim 10^{-11} \mathrm{~s}$, possibly inelastic scattering off magnetic ions. We note that, although inelastic scattering can explain the DOS broadening and gap reduction of the superconductor, spin-flip scattering provides additional channels for the Andreev current, introducing an uncertainty in spin-polarization measurements. ${ }^{28}$

We describe pair-breaking effects in the $\mathrm{Sn} /(\mathrm{Ga}, \mathrm{Mn}) \mathrm{As}$ contact using an empirical approach, ${ }^{29}$ wherein we account for inelastic scattering via an effective temperature $T^{*}$ and a reduced superconducting gap $\Delta$. Our approach provides a good description of the experimental data, as seen in Fig. 3. However, as we are unable to evaluate $\Delta$ and $T^{*}$ from first principles, our model leads to the uncertainty in $\Delta$ and a broadened DOS. This, in turn, yields a fairly large uncertainty in the extracted values of $P$ for $(\mathrm{Ga}, \mathrm{Mn}) \mathrm{As}, P$
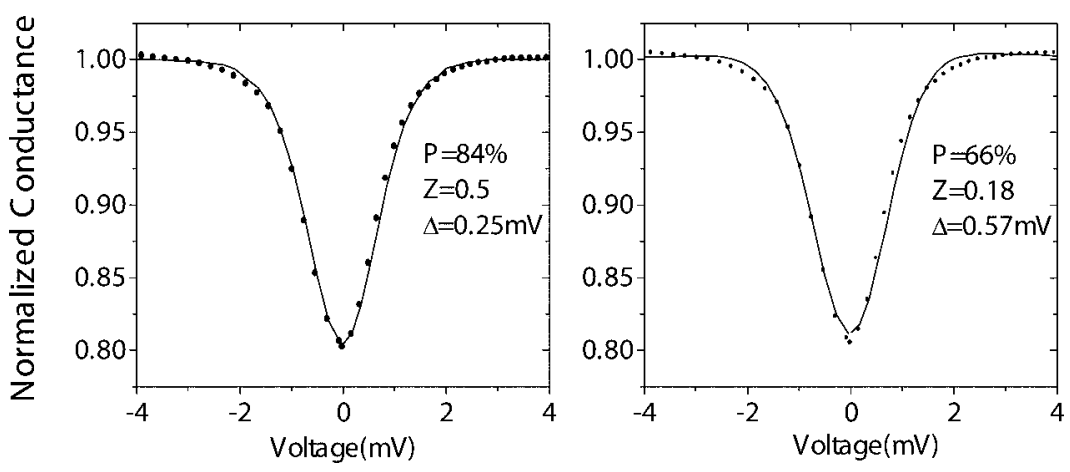

FIG. 3. Fits to the modified BTK model for $\mathrm{Sn} /(\mathrm{Ga}, \mathrm{Mn})$ As contact with $R_{c}=68 \Omega$ measured at $T=1.2 \mathrm{~K} . T^{*}=5.2 \mathrm{~K}$ was used for both fits. A reduced gap provides a better fit (left panel). The variation of $\Delta$ results in different $P$. 
$=83 \pm 17 \%$. We have observed a qualitatively similar-but quantitatively less significant-gap reduction and DOS broadening in ongoing PCAR studies of the ferromagnetic semiconductor $(\mathrm{Ga}, \mathrm{Mn}) \mathrm{Sb}$, whose mobility is between that of $(\mathrm{Ga}, \mathrm{Mn}) \mathrm{As}$ and $(\mathrm{In}, \mathrm{Mn}) \mathrm{Sb}^{30}{ }^{30}$ In that case, the accuracy in determining the spin polarization is significantly better, $P$ $=57 \pm 5 \% .{ }^{30}$ We note that, although we have a single critical temperature of Sn, $T_{c}=3.7 \mathrm{~K}$, the observed spectra are qualitatively quite similar to those in Ref. 7, suggesting that they may also be explained by the gap reduction and DOS broadening. Our observations are consistent with our conjecture that the PCAR measurements may suffer from inelastic scattering effects that enhance the uncertainty in measuring the spin polarization, particularly in highly spin-polarized materials characterized by unconventional transport mechanism, such as $(\mathrm{Ga}, \mathrm{Mn})$ As. This explanation is also in agreement with a recent experiment in superconductor and/or normal metal nanostructures, in which Pt impurities have been deliberately introduced at the superconductor-normal metal interface to enhance inelastic scattering. ${ }^{31}$

We thank R. A. Buhrman, A. A. Golubov, E. Demler, V. I. Fal'ko, I. I. Mazin, S. A. Wolf, and I. Zutic for useful discussions. The work was supported by the DARPA SpinS through ONR Grant No. N00014-02-1-0886 and NSF Career Grant No. 0239058 (B.N.), by ONR Grants No. N00014-991-0071, No. N00014-99-1-0716, and No. N00014-99-1-1093 (N.S), and by the DARPA SpinS and NSF-NIRT Grant No. DMR02-01519 (J.K.F.).
${ }^{1}$ A. G. Aronov, JETP Lett. 24, 32 (1976); Optical Orientation, edited by F. Meier and B. P. Zakharchenya (North-Holland, Amsterdam, 1984).

${ }^{2}$ T. Dietl, Semicond. Sci. Technol. 17, 377 (2002).

${ }^{3}$ H. Ohno, in Semiconductor Spintronics and Quantum Computation, edited by D. D. Awschalom, D. Loss, and N. Samarth (Springer-Verlag, Berlin, 2002), p. 1; N. Samarth, in Solid State Physics, edited by H. Ehrenreich and F. Spaepan (Academic Press, New York, 2004), Vol. 58, p. 1.

${ }^{4}$ K. C. Ku, S. J. Potashnik, R. F. Wang, S. H. Chun, P. Schiffer, N. Samarth, M. J. Seong, A. Mascarenhas, E. Johnston-Halperin, R. C. Myers, A. C. Gossard, and D. D. Awschalom, Appl. Phys. Lett. 82, 2302 (2003).

${ }^{5}$ K. W. Edmonds, P. Boguslawski, K. Y. Wang, R. P. Campion, S. N. Novikov, N. R. S. Farley, B. L. Gallagher, C. T. Foxon, M. Sawicki, T. Dietl, M. B. Nardelli, and J. Bernholc, Phys. Rev. Lett. 92, 037201 (2004).

${ }^{6}$ Y. Ohno, D. K. Young, B. Beschoten, F. Matsukura, H. Ohno, and D. D. Awschalom, Nature (London) 402, 790 (1999); M. Tanaka and Y. Higo, Phys. Rev. Lett. 87, 026602 (2001); S. H. Chun, S. J. Potashnik, K. C. Ku, P. Schiffer, and N. Samarth, Phys. Rev. B 66, 100408(R) (2002); R. Mattana, J.-M. George, H. Jaffrés, F. Nguyen Van Dau, A. Fert, B. Lépine, A. Guivarc'h, and G. Jézéquel, Phys. Rev. Lett. 90, 166601 (2003).

${ }^{7}$ J. G. Braden, J. S. Parker, P. Xiong, S. H. Chun, and N. Samarth, Phys. Rev. Lett. 91, 056602 (2003); 93, 169704 (2004).

${ }^{8}$ P. M. Tedrow and R. Meservey, Phys. Rep. 238, 173 (1994).

${ }^{9}$ K. Xia, P. J. Kelly, G. E. W. Bauer, and I. Turek, Phys. Rev. Lett. 89, 166603 (2002).

${ }^{10}$ M. J. M. de Jong and C. W. J. Beenakker, Phys. Rev. Lett. 74, 1657 (1995).

${ }^{11}$ R. J. Soulen, Jr., J. M. Byers, M. S. Osofsky, B. Nadgorny, T. Ambrose, S. F. Cheng, P. R. Broussard, C. T. Tanaka, J. Nowak, J. S. Moodera, A. Barry, and J. M. D. Coey, Science 282, 85 (1998).

${ }^{12}$ S. K. Upadhyay, A. Palanisami, R. N. Louie, and R. A. Buhrman, Phys. Rev. Lett. 81, 3247 (1998).

${ }^{13}$ I. Zutic, J. Fabian, and S. Das Sarma, Rev. Mod. Phys. 76, 323 (2004)

${ }^{14}$ M. B. Stone, K. C. Ku, S. J. Potashnik, B. L. Sheu, N. Samarth, and P. Schiffer, Appl. Phys. Lett. 83, 4568 (2003).
${ }^{15}$ C. H. Kant, A. T. Filip, H. J. M. Swagten, and W. J. M. de Jonge, Phys. Rev. Lett. 93,169703 (2004).

${ }^{16}$ G. E. Blonder, M. Tinkham, and T. M. Klapwijk, Phys. Rev. B 25, 4515 (1982)

${ }^{17}$ I. I. Mazin, A. A. Golubov, and B. Nadgorny, J. Appl. Phys. 89, 7576 (2001).

${ }^{18}$ R. P. Panguluri, B. Nadgorny, T. Wojtowicz, W. L. Lim, X. Liu, and J. K. Furdyna, Appl. Phys. Lett. 84, 4947 (2004).

${ }^{19}$ A. Kastalsky, A. W. Kleinsasser, L. H. Greene, R. Bhat, F. T. Milliken, and J. P. Harbison, Phys. Rev. Lett. 67, 3026 (1991).

${ }^{20}$ P. A. Barnes and A. Y. Choi, Appl. Phys. Lett. 33, 651 (1978).

${ }^{21}$ J. Konig, H.-H. Lin, and A. H. MacDonald, Phys. Rev. Lett. 84, 5628 (2000).

${ }^{22}$ P. M. Berciu and R. N. Bhatt, Phys. Rev. Lett. 87, 107203 (2001); A. Chattopadhyay, S. Das Sarma, and A. J. Millis, ibid. 87, 227202 (2001); E. J. Singley, R. Kawakami, D. D. Awschalom, and D. N. Basov, ibid. 89, 097203 (2002).

${ }^{23}$ R. P. Panguluri, G. Tsoi, B. Nadgorny, S. H. Chun, N. Samarth, and I. I. Mazin, Phys. Rev. B 68, 201307(R) (2003).

${ }^{24}$ I. Zutic and S. Das Sarma, Phys. Rev. B 60, R16322 (1999).

${ }^{25}$ Local magnetic fields from a ferromagnet may lead to suppression of superconductivity near the interface [e.g., Y. Miyoshi, Y. Bugoslavsky, and L. F. Cohen, cond-mat/0410264 (unpublished)]. This is possible if magnetic moment in (Ga,Mn)As has an out-of-plane component. However, due to a compressive strain, magnetization in $8 \% \mathrm{Mn}(\mathrm{Ga}, \mathrm{Mn})$ As epilayers is in plane.

${ }^{26}$ B. L. Altshuler and A. G. Aronov, Electron-Electron Interactions in Disordered Systems, edited by A. L. Efros and M. Pollak (Elsevier Science, Amsterdam, 1985).

${ }^{27}$ For a highly spin-polarized $(\mathrm{Ga}, \mathrm{Mn}) \mathrm{As}$, quasiparticles with $\varepsilon$ $\leqslant \Delta_{b}$ stay in the contact area even longer, until they find a partner of opposite spin to form a Cooper pair.

${ }^{28}$ Another possible mechanism of inelastic scattering is due to magnons, see G. Tkachov, E. McCann, and V. I. Fal'ko, Phys. Rev. B 65, 024519 (2001). This, however, should lead to a significant asymmetry of the $I-V$ characteristics, which has not been observed.

${ }^{29}$ R. C. Dynes, V. Narayanamurti, and J. P. Garno, Phys. Rev. Lett. 41, 1509 (1978).

${ }^{30}$ R. P. Panguluri et al. (unpublished).

${ }^{31}$ R. A. Buhrman (private communication). 